\title{
Choroidal Neovascularization Accompanied by Tractional Posterior Hyaloid Membrane Secondary to Candida Chorioretinitis
}

\author{
JaeWook Jung, YuCheol Kim*, Kwang Soo Kim \\ Department of Ophthalmology, Keimyung University School of Medicine, Dongsan Medical Center, Daegu, Republic of Korea \\ *Corresponding author: eyedr@dsmc.or.kr
}

Received April 16, 2015; Revised May 15, 2015; Accepted May 18, 2015

\begin{abstract}
A 62-year-old-male presented at our clinic with decreased vision and metamorphopsia(OD) for several days. He had received an anti-fungal treatment two months before for the treatment of endogenous candida choroiditis (OU). Fluorescein angiography and optical coherence tomography revealed subfoveal, juxtafoveal choroidal neovascularization and tractionalposterior hyaloidmembrane (OD). Pars planavitrectomy and epiretinal membrane removal was done, and twointravitrealbevacizumabinjections were administered. His visual symptoms were not improved and chorioretinal scar and fibrotic change were detected at fovea.
\end{abstract}

Keywords: candida retinitis, choroidal neovascularization, epiretinal membrane

Cite This Article: JaeWook Jung, YuCheol Kim, and Kwang Soo Kim, "Choroidal Neovascularization Accompanied by Tractional Posterior Hyaloid Membrane Secondary to Candida Chorioretinitis." American Journal of Medical Case Reports, vol. 3, no. 6 (2015): 167-169. doi: 10.12691/ajmcr-3-6-5.

\section{Introduction}

Candidiasis is a common cause of endogenous endophthalmitis, Candida albicans is the single most common pathogen that causes fungal endophthalmitis. [1] Hematogenous dissemination of C. albicans may cause choroiditis, retinitis and vitritis. [2] Systemic antifungal therapy has proved successful in treating candida endophthalmitis, but in severe cases, vitrectomy with intravitreal antifungal agent injection is necessary. $[3,4,5]$ However, choroidal neovascularization (CNV) or tractionalepiretinal membrane may follow successful treatment of candida endophthalmitis with subsequent visual loss. [6,7] A PubMed and Google search revealed no reported cases of secondary CNV accompanied by tractional posterior hyaloid membrane secondary to candida endophthalmitis, using spectral domain optical coherence tomography (SD-OCT). We report a case of CNV and tractional membrane secondary to Candida chorioretinitis using SD-OCT.

\section{Case Presentation}

A 62-year-old male presented at our clinic with decreased vision (OU) for several days. He was hospitalized for alcoholic pancreatitis. He had a Hickman catheter placed for parenteral nutrition and C. albicans was identified in his blood culture. Best corrected visual acuity (BCVA) was 5/200 (OD) and 20/200 (OS). Fundus examination revealed multiple round yellow-white lesions with diagnosis of endogenous Candida chorioretinitis.

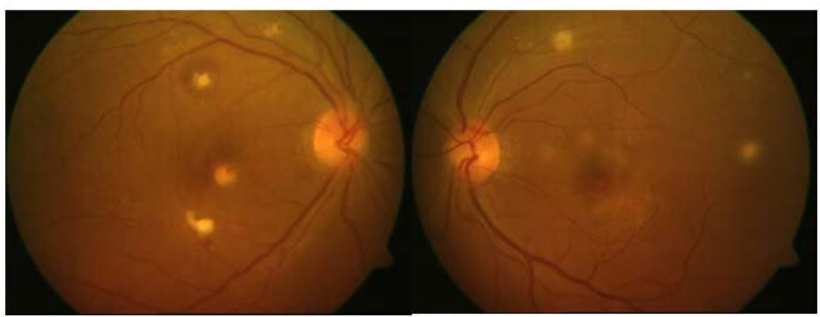

(A)

(B)

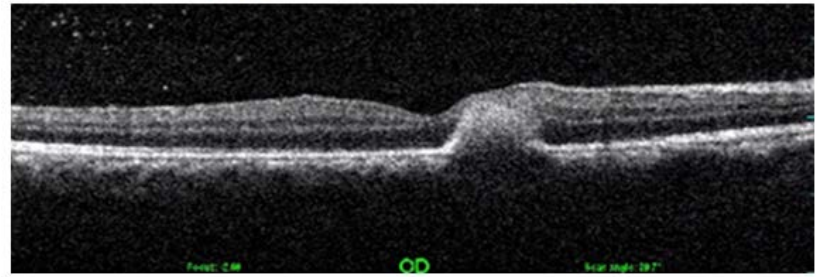

(C)

Figure 1. (A) and (B) Fundus photographs show multifocal yellow-white chorioretinal infiltration with suspected pin-point retinal hemorrhage.(C) Optical coherence tomography reveals retinal and subretinalhyperreflective lesion at fovea and juxtafovea

Intravenous fluconazole $200 \mathrm{mg}$ /day for one month was administered. After the treatment, active inflammation subsided with chorioretinal scar (Figure 1). One month later he complained of decreased vision (OD). BCVA (OD) was decreased to 10/200, compared with 20/30 (OS). Fundus examination (OD) revealed tractional membrane and subretinal hemorrhage around chorioretinal scar lesions (Figure 2A). OCT showed hyperreflectivechorioretinal 
lesion with posterior hyaloid membrane traction (Figure 2B). Fluorescein angiographic features showed slight leakage at fovea in the late phase (Figure 2C). On the basis of surrounding subretinal hemorrhage, fluorescein leakage and OCT findings, secondary CNV was diagnosed. Intravitrealbevacizumab injection $(1.25 \mathrm{mg} / 0.05 \mathrm{ml})$ was performed. No change was evident. The patient underwent pars planavitrectomy with epiretinal and internal limiting membrane removal. One month after the surgery (Figure 3), intravitrealbevacizumabwas injected again. One month after the second injection, subretinal fluid was absorbed (Figure 4). Since then, it has been stable for one year.

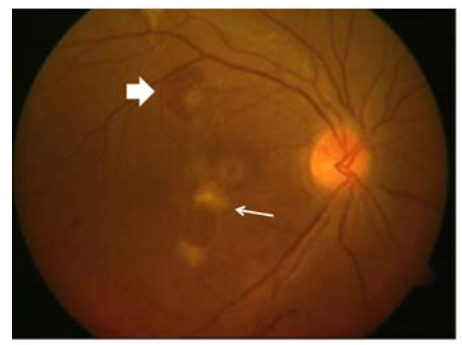

(A)

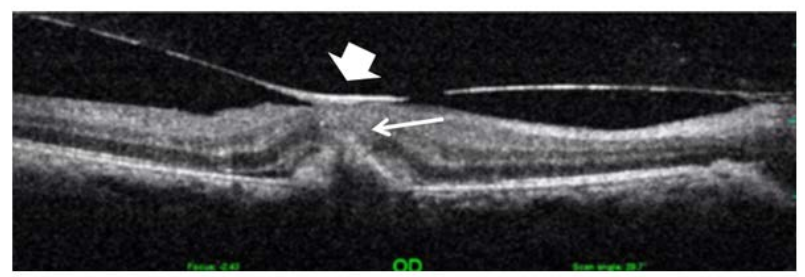

(B)

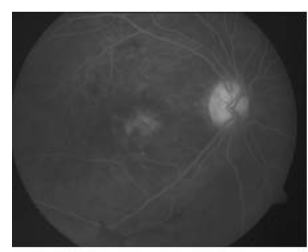

(C)

Figure 2. (A) Fundus photograph shows chorioretinal scars with surrounding retinal hemorrhage (thick arrow) and tractional membrane (thin arrow). (B)Optical coherence tomography shows fibrovascular complex with minimal subretinal fluid and tractional membrane (thick arrow) connected withretinal scar (thin arrow). (C) Fluorescein angiogram demonstrates minimal leakage in late phase

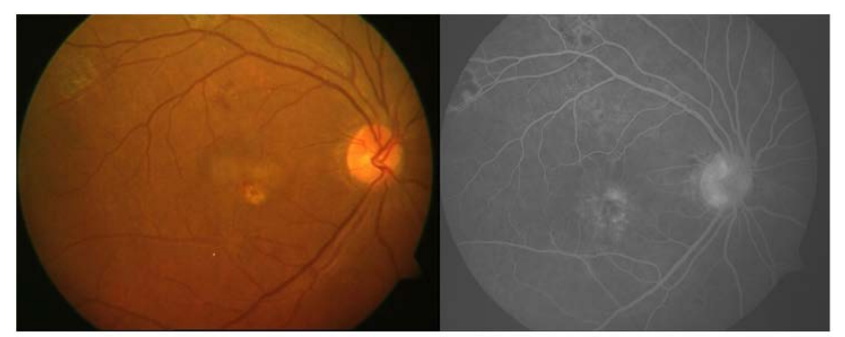

(A)

(B)

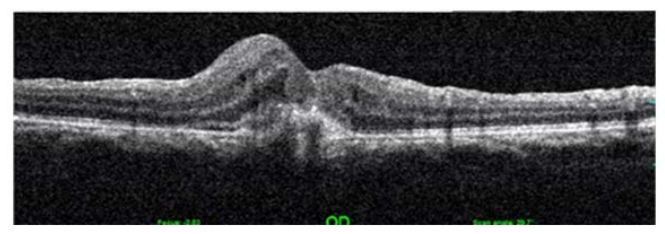

(C)

Figure 3. (A) fundus photography and (B) fluorescein angiography, after the surgery and bevacizumab injection, (C) Optical coherence tomography reveals that the tractional membrane was well removed

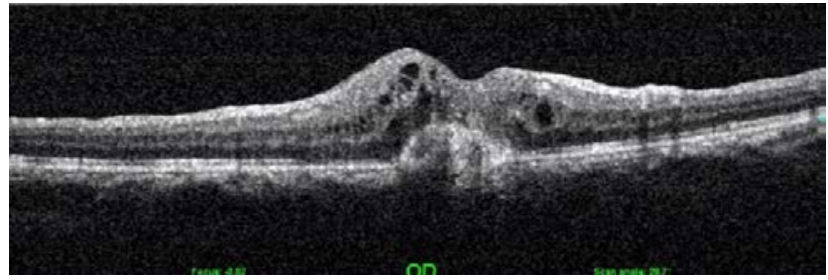

Figure 4. Optical coherence tomography after surgery and twice bevacizumab injection shows chorioretinal scar without subretinal fluid

\section{Discussion}

Candida infection can lead to visual loss through several mechanisms. These include direct or ischemic damage to the macula or the optic nerve, epiretinal membrane formation, vitreoretinal traction, or retinal detachment. [6] Candidal lesions are found in the inner choroid and may break through Bruch's membrane to form subretinalabcesses or involve full-thickness retina, and it is not surprising to see subretinal neovascularization, full-thickness retinal scar, and epiretinal membranes distorting the retinal surface even after successfully treatment of Candida retinitis. [7] Also, theepiretinal membrane may result from inflammation and vitreous traction associated with the candida endophthalmitis.

Retinal circulatory failure with inflammation may cause formation of the proliferative tissue. [6] Only a few cases of epiretinal membrane secondary to candida have been previously reported $[7,8]$.

Bebbe et al. [3] reported subretinal neovascularization membrane as a complication of endogenous candida endophthalmitis. It is presumed that CNV and tractional membrane induce and aggravate each other. The diagnosis of CNV may be difficult when tractionalepiretinal membrane and CNV exist together. In this case, it was not easy to differentiate the CNV from chorioretinal scar because leakage was not clear on fluoroangiographic examination. However, subretinal hemorrhage was the clue for the diagnosis of CNV.

Jampol et al. [6] suggested that laser photocoagulation and surgical excision of the neovascular complex may be good option in selected cases with CNV secondary to candida chorioretinitis. Tedeshci et al. [9] reported that photodynamic therapy is safe and useful to arrest CNV progression in the fovealresion for secondary choroidal neovascularization in candida endophthalmitis. Sheu [10] reported a good response to one dose of intravitrealranibizumab for choroidal neovascularization without recurrence or complication during 10 month follow- up. Lee et al. [11] suggested that intravitreal bevacizumab injection can effectively treat CNV and improve visual symptoms for the treatment of juxtafoveal CNV associated with candida choroiditis. However, in the case presented here, bevacizumab alone was not effective and it is presumed to be because of the tractional membrane. Lee et al. [12] suggested that CNV with vitreomacular adhesion could be refractory to antivascular endothelium growth factor. Vitrectomy and membranectomy can be expected to be effective in treating CNV. However, the half-life of the intravitreal injection in vitrectomized eye would not be as long as in non-vitrectomized eyes. 
This case report shows CNV and tractionalhyaloid membrane secondary to Candida chorioretinitis using serial SD-OCT images and suggests to pay close attention to differentiating secondary $\mathrm{CNV}$ and retinal scar especially when accompanied by tractional membrane.

\section{Interest and Support}

No author has a financial or proprietary interest in any material or method mentioned.

There was no financial support for this study

\section{References}

[1] Parke DW, 2nd, Jones DB, Gentry LO. Endogenous endophthalmitis among patients with candidemia. Ophthalmology. 1982;89(7):789-796.

[2] Donahue SP, Greven CM, Zuravleff JJ, et al. Intraocular candidiasis in patients with candidemia. Clinical implications derived from a prospective multicenter study. Ophthalmology. 1994;101(7):1302-1309.

[3] Beebe WE, Kirkland C, Price J. A subretinal neovascular membrane as a complication of endogenous Candida endophthalmitis. Ann Ophthalmol. 1987;19(6):207-209.
[4] Stern GA, Fetkenhour CL, O'Grady RB. Intravitreal amphotericin B treatment of Candida endophthamitis. Arch Ophthalmol. 1977;95(1):89-93.

[5] Gilbert CM, Novak MA. Successful treatment of postoperative Candida endophthalmitis in an eye with an intraocular lens implant. Am J Ophthalmol. 1984;97(5):593-595.

[6] Jampol LM, Sung J, Walker JD, et al. Choroidal neovascularization secondary to Candida albicans chorioretinitis. Am J Ophthalmol. 1996;121(6):643-649.

[7] McDonald HR, De Bustros S, Sipperley JO. Vitrectomy for epiretinal membrane with Candida chorioretinitis. Ophthalmology. 1990;97(4):466-469.

[8] Naoi N, Sawada A. Effect of vitrectomy on epiretinal membranes after endogenous fungal endophthalmitis. Jpn J Ophthalmol. 1996;40(3):434-438.

[9] Tedeschi M, Varano M, Schiano Lomoriello D, Scassa C, Parisi V. Photodynamic therapy outcomes in a case of macular choroidal neovascularization secondary to Candida endophthalmitis. Eur $J$ Ophthalmol. 2007;17(1):124-127.

[10] Sheu SJ. Intravitreal ranibizumab for the treatment of choroidal neovascularization secondary to endogenous endophthalmitis. Kaohsiung J Med Sci. 2009;25(11):617-621.

[11] Lee SH, Lee JW, Shin YU, Lee BR. Intravitreal bevacizumab injection for the treatment of choroidal neovascularization secondary to candida chorioretinitis. J Korean Ophthalmol Soc. 2014;55(7):1106-1110.

[12] Lee SJ, Koh HJ. Effects of vitreomacular adhesion on antivascular endothelial growth factor treatment for exudative agerelated macular degeneration. Ophthalmology. 2011;118(1):101110. 